PROCEEDINGS OF THE

AMERICAN MATHEMATICAL SOCIETY

Volume 131, Number 6, Pages 1817-1827

S 0002-9939(02)06716-3

Article electronically published on October 1, 2002

\title{
THE PRODUCT OF A NONSYMMETRIC JACK POLYNOMIAL WITH A LINEAR FUNCTION
}

\author{
DAN MARSHALL
}

(Communicated by John R. Stembridge)

\begin{abstract}
In this paper a decomposition in terms of the nonsymmetric Jack polynomials is given for the product of any nonsymmetric Jack polynomial $E_{\eta}(z)$ with $z_{i}$. This decomposition generalises a recurrence formula satisfied by single variable orthogonal polynomials on the unit circle. The decomposition also allows the evaluation of the generalised binomial coefficients $\left(\begin{array}{l}\eta \\ \nu\end{array}\right)$ associated with the nonsymmetric Jack polynomials for $|\eta|=|\nu|+1$.
\end{abstract}

\section{INTRODUCTION}

Let $\kappa:=\left(\kappa_{1}, \ldots, \kappa_{n}\right)$ be a partition. The modulus of $\kappa$ is defined by $|\kappa|:=$ $\sum_{i=1}^{n} \kappa_{i}$. The symmetric Jack polynomial $P_{\kappa}:=P_{\kappa}(z ; \alpha)$ is a function of $n$ variables $z=\left(z_{1}, \ldots, z_{n}\right)$ and has coefficients in the field $\mathbb{Q}(\alpha)$ of rational functions of the indeterminant $\alpha$. It can be defined as the unique symmetric eigenfunction of the differential operator

$$
D_{2}(\alpha):=\sum_{j=1}^{n} z_{j}^{2} \frac{\partial^{2}}{\partial z_{j}^{2}}+\frac{2}{\alpha} \sum_{\substack{1 \leq j, k \leq n \\ j \neq k}} \frac{z_{j}^{2}}{z_{j}-z_{k}} \frac{\partial}{\partial z_{j}}
$$

that is of the form

$$
P_{\kappa}(z ; \alpha)=m_{\kappa}(z)+\sum_{\lambda<\kappa} u_{\kappa \lambda} m_{\lambda}(z)
$$

In (1.2), $m_{\kappa}(z)$ is the monomial symmetric function in the variables $z_{1}, \ldots, z_{n}$. The ordering $<$ is the dominance ordering on partitions having the same modulus. It is defined by $\lambda<\kappa$ iff $\lambda \neq \kappa$ and $\sum_{i=1}^{p}\left(\kappa_{i}-\lambda_{i}\right) \geq 0$ for all $1 \leq p \leq n$.

The symmetric Jack polynomials are a class of multi-variable orthogonal polynomials on the unit circle. They possess a number of important properties including analogues of properties held by classical single variable orthogonal polynomials. In particular, they satisfy the Pieri formula (1.3) [13].

Received by the editors May 14, 2001 and, in revised form, December 7, 2001, January 11, 2002, and January 22, 2002.

2000 Mathematics Subject Classification. Primary 33C45; Secondary 05A10.

Key words and phrases. Jack polynomials, Pieri formula, generalized binomial coefficients.

The author thanks Peter Forrester for useful discussions and for bringing to his attention the paper by Knop and Sahi, and an anonymous referee for helpful comments. This work was supported by an Australian Postgraduate Award. 


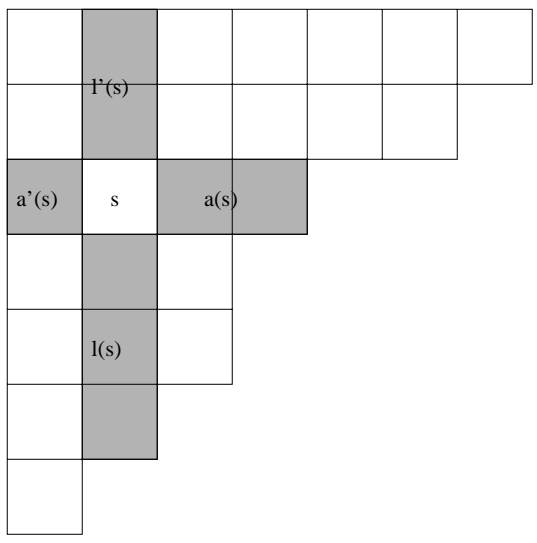

FiguRE 1. The Young diagram of $(7,6,4,3,3,2,1)$

Presenting the Pieri formula requires some notation. The Young diagram of $\kappa$ is the set $\operatorname{diag}(\kappa):=\left\{(i, j) \mid 1 \leq j \leq \kappa_{i}, 1 \leq i \leq n\right\}$ which is drawn with $i$ increasing from top to bottom and $j$ from left to right. Each element of $\operatorname{diag}(\kappa)$ is called a node of $\kappa$. If $\operatorname{diag}(\kappa) \subset \operatorname{diag}(\lambda)$, then the skew diagram $\lambda / \kappa$ is the set $\operatorname{diag}(\lambda) / \operatorname{diag}(\kappa)$. The skew diagram $\lambda / \kappa$ is said to be a vertical $r$-strip if it consists of $r$ nodes, all of which are in distinct rows. The Pieri formula is

$$
e_{r}(z) P_{\kappa}(z ; \alpha)=\sum_{\lambda: \lambda / \kappa \text { is a vertical } r \text {-strip }} \psi_{\lambda / \kappa}(\alpha) P_{\lambda}(z ; \alpha),
$$

where

$$
e_{r}(z)=\sum_{1 \leq i_{1}<\cdots<i_{r} \leq n} z_{i_{1}} \cdots z_{i_{r}}
$$

is the $r$ th elementary symmetric function. For each node $s=(i, j)$ of $\kappa$, the number of points to the right, left, below and above $s$ are its arm length $a_{\kappa}(s):=\kappa_{i}-j$, arm colength $a_{\kappa}^{\prime}(s):=a_{\kappa}^{\prime}(j):=j-1$, leg length $l_{\kappa}(s):=\#\left\{k \mid k>i, j \leq \kappa_{k}\right\}$, and leg colength $l_{\kappa}^{\prime}(s):=l_{\kappa}^{\prime}(i):=i-1$. Let $\chi(\lambda / \kappa)$ denote the set of all nodes $(i, j) \in \operatorname{diag}(\lambda)$ such that $\kappa_{i}=\lambda_{i}$ and $l_{\kappa}(1, j)<l_{\lambda}(1, j)$. Then

$$
\psi_{\lambda / \kappa}(\alpha):=\prod_{s \in \chi(\lambda / \kappa)} \frac{\left(\alpha a_{\lambda}(s)+l_{\lambda}(s)+1\right)\left(\alpha\left(a_{\kappa}(s)+1\right)+l_{\kappa}(s)\right)}{\left(\alpha a_{\kappa}(s)+l_{\kappa}(s)+1\right)\left(\alpha\left(a_{\lambda}(s)+1\right)+l_{\lambda}(s)\right)} .
$$

When $n=1$, the Pieri formula reduces to

$$
z P_{k}(z ; \alpha)=P_{k+1}(z ; \alpha) .
$$

This is a special case of a recurrence formula which applies to any class of single variable orthogonal polynomials on the unit circle [14, p. 291].

The symmetric Jack polynomials are closely related to a more fundamental class of orthogonal polynomials called the nonsymmetric Jack polynomials [8]. These polynomials can be usefully employed to illuminate and simplify the theory of their symmetric counterparts [2]. They also possess many interesting properties in their own right. In this paper a decomposition in terms of the nonsymmetric Jack polynomials is given for the product of any nonsymmetric Jack polynomial with 
$z_{i}$. The existence of this decomposition was conjectured by Cherednik [3]. Like the Pieri formula (1.3) the decomposition reduces to (1.6) when $n=1$.

The derivation of this formula is similar to Knop and Sahi's derivation of (1.3) [6]. Knop and Sahi's derivation exploits the theory of the recently introduced interpolated symmetric Jack polynomials [9. We shall proceed along similar lines using the interpolated nonsymmetric Jack polynomials which are nonsymmetric analogues of these polynomials 10,5 .

The interpolated nonsymmetric Jack polynomials are closely related to a class of generalised binomial coefficients $\left(\begin{array}{l}\eta \\ \nu\end{array}\right)$ indexed by pairs of compositions [1]. As a corollary we give an evaluation of these coefficients for $|\eta|=|\nu|+1$.

\section{Preliminaries}

Let $\eta:=\left(\eta_{1}, \ldots, \eta_{n}\right)$ be a composition, that is, an $n$-tuple of non-negative integers. The modulus and Young diagram of $\eta$ receive the same definition as that given in Section 1 for partitions. The partition $\eta^{+}$is defined as the unique partition obtained by permuting the components of $\eta$. The nonsymmetric Jack polynomial $E_{\eta}$ can be defined as the unique polynomial of the form

$$
E_{\eta}(z ; \alpha)=z^{\eta}+\sum_{\mu \prec \eta} a_{\eta \mu} z^{\mu}
$$

that is, an eigenfunction of each of the Cherednik operators

$\xi_{i}:=\alpha z_{i} \frac{\partial}{\partial z_{i}}+\sum_{p<i} \frac{z_{i}}{z_{i}-z_{p}}\left(1-s_{i p}\right)+\sum_{p>i} \frac{z_{p}}{z_{i}-z_{p}}\left(1-s_{i p}\right)+1-i, \quad i=1, \ldots, n$.

In (2.1), $z^{\eta}:=z_{1}^{\eta_{1}} \cdots z_{n}^{\eta_{n}}$ and $\prec$ is a partial ordering on compositions having the same modulus. The ordering $\prec$ is defined by $\nu \prec \eta$ iff

$$
\text { either } \nu^{+}<\eta^{+} \text {, or else } \nu^{+}=\eta^{+} \text {and } \nu<\eta \text {. }
$$

The ordering $<$ in (2.3) is the dominance ordering on compositions having the same modulus and receiving the same definition as the dominance ordering on partitions. The eigenvalue of $E_{\eta}$ with respect to $\xi_{i}$ is

$$
\bar{\eta}_{i}:=\alpha \eta_{i}-l_{\eta}^{\prime}(i),
$$

where $l_{\eta}^{\prime}(i)$ is the leg colength of $s=(i, j)$ with respect to the composition $\eta$. The definition of leg colength given in Section 1 has to be extended to apply to compositions. The extension is [1]

$$
l_{\eta}^{\prime}(i):=\#\left\{k \mid k<i, \eta_{k} \geq \eta_{i}\right\}+\#\left\{k \mid k>i, \eta_{k}>\eta_{i}\right\} .
$$

Arm length, arm colength, and leg length are also extended. Arm length and colength receive the same definition as in Section 1, Leg length has definition [7]

$$
l_{\eta}(s)=\#\left\{k \mid k<i, j \leq \eta_{k}+1 \leq \eta_{i}\right\}+\#\left\{k \mid k>i, j \leq \eta_{k} \leq \eta_{i}\right\} .
$$

A diagrammatic computation of (2.5) and (2.6) is undertaken in Figure 2.

The interpolated Jack polynomials are generalisations of the classical shifted polynomials

$$
(-1)^{p}(-z)_{p}:=z(z-1) \cdots(z-p+1) .
$$




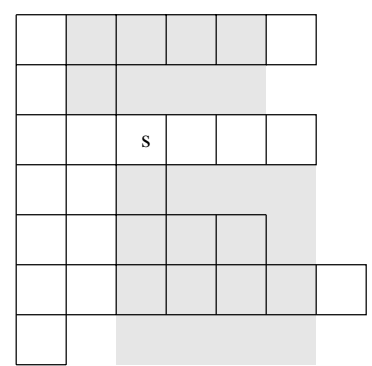

$1(\mathrm{~s})=3$

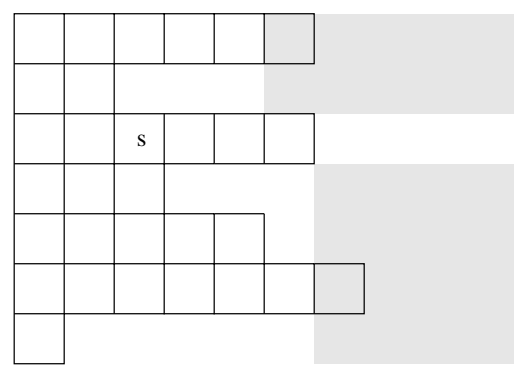

$l^{\prime}(s)=2$

FiguRe 2. In the left-hand side diagram the leg length is calculated as the number of rows which have shaded boxes and no unshaded boxes to the right of the shaded boxes. In the right-hand side diagram the leg colength is calculated as the number of rows which have any box shaded.

The interpolated nonsymmetric Jack polynomial $E_{\eta}^{*}$ can be defined as the unique polynomial satisfying:

$$
\begin{aligned}
& \text { i): } E_{\eta}^{*}\left(\frac{\bar{\nu}}{\alpha}\right)=0, \quad|\nu| \leq|\eta|, \nu \neq \eta ; \\
& \text { ii): } E_{\eta}^{*}\left(\frac{\bar{\eta}}{\alpha}\right) \neq 0 ; \\
& \text { iii) }: E_{\eta}^{*}(z)=z^{\eta}+\sum_{\substack{|\nu| \leq|\eta| \\
\nu \neq \eta}} b_{\eta \nu} z^{\nu} .
\end{aligned}
$$

Two properties of the polynomials $E_{\eta}^{*}$ are given by the following two lemmas.

Lemma 2.1 ([5]). If $\eta_{n}=0$, then

$$
E_{\eta}^{*}\left(z_{1}, \ldots, z_{n-1},-\frac{n-1}{\alpha}\right)=E_{\eta^{\prime}}^{*}\left(z_{1}, \ldots, z_{n-1}\right),
$$

where $\eta^{\prime}:=\left(\eta_{1}, \ldots, \eta_{n-1}\right)$.

Lemma $2.2([5])$. Define an operator $\Delta$ so that for any polynomial $f(z)$,

$$
\Delta f\left(z_{1}, \ldots, z_{n}\right):=f\left(z_{n}-1, z_{1}, \ldots, z_{n-1}\right) .
$$

We have

$$
E_{\Phi \eta}^{*}(z)=\left(z_{n}+\frac{n-1}{\alpha}\right) \Delta E_{\eta}^{*}(z),
$$

where $\Phi \eta:=\left(\eta_{2}, \ldots, \eta_{n}, \eta_{1}+1\right)$.

The interpolated Jack polynomial $E_{\eta}^{*}$ is defined to vanish for all $z=\frac{\bar{\nu}}{\alpha}$ where $|\nu| \leq|\eta|, \nu \neq \eta$. It turns out that $E_{\eta}^{*}$ vanishes on a larger domain. To state this domain requires introducing a further partial ordering on compositions. For compositions $\nu, \eta$ we say that $\nu \preceq^{\prime} \eta$ iff there exists a permutation $\pi$ such that

$$
\nu_{i}<\eta_{\pi(i)} \text { if } i<\pi(i) \text {, and } \nu_{i} \leq \eta_{\pi(i)} \text { if } i \geq \pi(i) .
$$

This partial order reduces to the usual inclusion relation among diagrams when restricted to partitions.

Proposition 2.3 ([5]). If $\eta \npreceq^{\prime} \mu$, then $E_{\eta}^{*}\left(\frac{\bar{\mu}}{\alpha}\right)=0$. 
The minimal elements lying above $\eta$ according to $\prec^{\prime}$ play an important role in the next section. Let $I=\left\{i_{1}, \ldots, i_{r}\right\}$ where $i_{1}<\ldots<i_{r}$. We define the composition $c_{I}(\eta):=\mu$ by:

$$
\begin{aligned}
& \text { i): } \mu_{i_{j}}=\eta_{i_{j+1}}, \quad j=1, \ldots, r-1 ; \\
& \text { ii): } \mu_{i_{r}}=\eta_{i_{1}}+1 ; \\
& \text { iii): } \mu_{i}=\eta_{i}, \quad i \notin I .
\end{aligned}
$$

For example, if

$$
\eta:=\left(\ldots, \eta_{i_{1}}, \ldots, \eta_{i_{2}}, \ldots, \eta_{i_{3}}, \ldots, \eta_{i_{r-1}}, \ldots, \eta_{i_{r}}, \ldots\right),
$$

then

$$
c_{I}(\eta):=\left(\ldots, \eta_{i_{2}}, \ldots, \eta_{i_{3}}, \ldots, \eta_{i_{4}}, \ldots, \eta_{i_{r}}, \ldots, \eta_{i_{1}}+1, \ldots\right) .
$$

Clearly $\eta \prec^{\prime} c_{I}(\eta)$. The following gives the converse.

Lemma 2.4 ([5]). If $\eta \prec^{\prime} \mu$, then there exists an $I$ such that $c_{I}(\eta) \preceq^{\prime} \mu$.

Define the operator $\sigma_{i}$ by

$$
\sigma_{i}:=s_{i}+\frac{1}{\alpha} \frac{1-s_{i}}{z_{i}-z_{i+1}}, \quad i=1, \ldots, n-1,
$$

where $s_{i}$ is the transposition operator that acts on functions of $z:=\left(z_{1}, \ldots, z_{n}\right)$ by interchanging the variables $z_{i}$ and $z_{i+1}$. The interpolated Jack polynomial $E_{\eta}^{*}$ is a simultaneous eigenfunction of the family of operators

$$
\tilde{\Xi}_{i}:=z_{i}-\sigma_{i} \cdots \sigma_{n-1}\left(z_{n}+\frac{n-1}{\alpha}\right) \Delta \sigma_{1} \cdots \sigma_{i-1}, \quad i=1, \ldots, n-1 .
$$

These operators can be used to show that the interpolated Jack polynomials are triangular with respect to the nonsymmetric Jack polynomials. For each polynomial $E_{\eta}^{*}$ we can write

$$
E_{\eta}^{*}(z)=E_{\eta}(z)+\sum_{|\nu|<|\eta|} \tilde{a}_{\eta \nu} E_{\nu}(z)
$$

for coefficients $\tilde{a}_{\eta \nu}$. This decomposition shows that the top homogeneous component of any interpolated Jack polynomial $E_{\eta}^{*}(z)$ is the Jack polynomial $E_{\eta}(z)$. Using (2.14) we can define an isomorphism $\Psi$ mapping each Jack polynomial $E_{\eta}$ to its corresponding interpolated polynomial $E_{\eta}^{*}$. This isomorphism allows us to state the following important inversion formula.

Proposition 2.5 ([5]). Define

$$
\tilde{Z}_{i}:=\sigma_{i} \cdots \sigma_{n-1}\left(z_{n}+\frac{n-1}{\alpha}\right) \Delta \sigma_{1} \cdots \sigma_{i-1}, \quad i=1, \ldots, n-1 .
$$

We have

$$
\tilde{Z}_{i} \Psi=\Psi z_{i}
$$

\section{A JaCK POLYNOMIAL DECOMPOSITION}

We first give an evaluation formula for the interpolated Jack polynomials.

Proposition 3.1. We have

$$
E_{\eta}^{*}\left(\frac{\bar{\eta}}{\alpha}\right)=\frac{d_{\eta}^{\prime}}{\alpha^{|\eta|}}
$$


where

$$
d_{\eta}^{\prime}:=\prod_{s \in \operatorname{diag}(\eta)}\left(\alpha\left(a_{\eta}(s)+1\right)+l_{\eta}(s)\right) .
$$

Proof. We proceed by induction on $n+|\eta|$. First suppose that $\eta_{n}=0$. Then $\left(\frac{\bar{\eta}}{\alpha}\right)_{n}=-\frac{n-1}{\alpha}$. Applying Lemma 2.1 and the inductive hypothesis we obtain

$$
E_{\eta}^{*}\left(\frac{\bar{\eta}}{\alpha}\right)=E_{\mu}^{*}\left(\frac{\bar{\mu}}{\alpha}\right)=\frac{1}{\alpha^{|\eta|}} \prod_{s \in \operatorname{diag}(\mu)}\left(\alpha\left(a_{\mu}(s)+1\right)+l_{\mu}(s)\right)
$$

where $\mu:=\left(\eta_{1}, \ldots, \eta_{n-1}\right)$. We obtain (3.1) since $a_{\eta}(s)=a_{\mu}(s)$ and $l_{\eta}(s)=l_{\mu}(s)$ for each $s \in \operatorname{diag}(\mu)=\operatorname{diag}(\eta)$.

Now suppose that $\eta_{n}>0$. Applying Lemma 2.2 gives

$$
E_{\eta}^{*}(z)=\left(z_{n}+\frac{n-1}{\alpha}\right) E_{\mu}^{*}\left(z_{n}-1, z_{1}, \ldots, z_{n-1}\right)
$$

where $\mu:=\Phi^{-1} \eta$. Evaluating (3.4) at $z=\frac{\bar{\eta}}{\alpha}$ we obtain

$$
\begin{aligned}
E_{\eta}^{*}\left(\frac{\bar{\eta}}{\alpha}\right) & =\left(\eta_{n}+\frac{n-1-l_{\eta}^{\prime}(n)}{\alpha}\right) E_{\mu}^{*}\left(\frac{\bar{\mu}}{\alpha}\right) \\
& =\frac{1}{\alpha^{|\eta|}}\left(\alpha\left(a_{\eta}(n, 1)+1\right)+l_{\eta}(n, 1)\right) \prod_{s \in \operatorname{diag}(\mu)}\left(\alpha\left(a_{\mu}(s)+1\right)+l_{\mu}(s)\right) .
\end{aligned}
$$

In the second line we have used the inductive hypothesis together with the identities $\eta_{n}=a_{\eta}(n, 1)+1$ and $l_{\eta}(n, 1)=n-1-l_{\eta}^{\prime}(n)$. Now consider the bijection $\psi$ : $\operatorname{diag}(\mu) \mapsto \operatorname{diag}(\Phi \mu) /\{(n, 1)\}$ where

$$
\psi(i, j):= \begin{cases}(n, j+1) & \text { if } i=1 \\ (i-1, j) & \text { if } i>1\end{cases}
$$

We obtain (3.1) by noting that $a_{\mu}(s)=a_{\Phi \mu}(\psi s)$ and $l_{\mu}(s)=l_{\Phi \mu}(\psi s)$ for each $s \in \operatorname{diag}(\mu)$.

Proposition 3.2. The action of $\tilde{Z}_{i}$ on $f(z)$ is given by

$$
\tilde{Z}_{i} f(z)=\sum_{\substack{I \subseteq\{1, \ldots, n\} \\ i \in I}} a_{I}^{(i)}(z) f\left(c_{I}^{-1}(z)\right) .
$$

The rational function $a_{I}^{(i)}(z)$ can be expressed as

$$
a_{I}^{(i)}(z)=\chi_{I}^{(i)}(z) A_{I}(z) B_{I}(z)
$$


where

$$
\begin{aligned}
& A_{I}(z):= \frac{1}{\alpha^{s-1}\left(z_{t_{s}}-z_{t_{1}}-1\right)} \prod_{u=1}^{s-1} \frac{1}{\left(z_{t_{u}}-z_{t_{u+1}}\right)}, \\
& B_{I}(z):=\left(\prod_{j=t_{s}+1}^{n} \frac{z_{t_{s}}-z_{j}-\frac{1}{\alpha}}{z_{t_{s}}-z_{j}}\right)\left(z_{t_{s}}+\frac{n-1}{\alpha}\right) \\
& \times\left(\prod_{j=1}^{t_{1}-1} \frac{z_{t_{s}}-1-z_{j}-\frac{1}{\alpha}}{z_{t_{s}}-1-z_{j}}\right)\left(\prod_{u=1}^{s-1} \prod_{j=t_{u}+1}^{t_{u+1}-1} \frac{z_{t_{u}}-z_{j}-\frac{1}{\alpha}}{z_{t_{u}}-z_{j}}\right), \\
& \chi_{I}^{(i)}(z):= \begin{cases}z_{t_{k-1}}-z_{i} & \text { if } i=t_{k}, k \in\{2,3, \ldots, s\}, \\
z_{t_{s}}-z_{i}-1 & \text { if } i=t_{1},\end{cases}
\end{aligned}
$$

and $I=\left\{t_{1}, \ldots, t_{s}\right\}, 1 \leq t_{1}<\cdots<t_{s} \leq n$.

Proof. The action of $\sigma_{i}$ on any polynomial $f(z)$ is given by

$$
\sigma_{i} f(z)=\mathcal{A}_{i} f(z)+\mathcal{B}_{i} f(z)
$$

where

$$
\mathcal{A}_{i}:=\frac{1}{\alpha\left(z_{i}-z_{i+1}\right)}, \quad \quad \mathcal{B}_{i}:=\frac{z_{i}-z_{i+1}-\frac{1}{\alpha}}{z_{i}-z_{i+1}} s_{i} .
$$

Given definition (2.15) of $\tilde{Z}_{i}$ we can write

$$
\tilde{Z}_{i}=\sum_{\substack{1 \leq t_{1}<\cdots<t_{s} \leq n \\ i=t_{k}}} U_{t_{k}} \cdots U_{t_{s}} U_{t_{1}} \cdots U_{t_{k-1}}
$$

where

$$
\begin{aligned}
& U_{t_{j}}:=\mathcal{A}_{t_{j}} \mathcal{B}_{t_{j}+1} \cdots \mathcal{B}_{t_{j+1}-1}, \quad 1 \leq j<k, \\
& U_{t_{s}}:=\mathcal{A}_{t_{s}-1} \mathcal{B}_{t_{s}} \cdots \mathcal{B}_{n-1}\left(z_{n}+\frac{n-1}{\alpha}\right) \Delta \mathcal{B}_{1} \cdots \mathcal{B}_{t_{1}-1}, \\
& U_{t_{j}}:=\mathcal{A}_{t_{j-1}} \mathcal{B}_{t_{j}} \cdots \mathcal{B}_{t_{j+1}-2}, \quad k<j<s
\end{aligned}
$$

and

$$
U_{i}:= \begin{cases}\mathcal{B}_{i} \cdots \mathcal{B}_{t_{k+1}-2} & \text { if } i \neq s, \\ \mathcal{B}_{i} \cdots \mathcal{B}_{n-1}\left(z_{n}+\frac{n-1}{\alpha}\right) \Delta \mathcal{B}_{1} \cdots \mathcal{B}_{t_{1}-1} & \text { if } i=s .\end{cases}
$$

By applying (3.13) to $f(z)$ we can directly compute (3.7).

We are now in a position to derive an expansion of $z_{i} E_{\eta}$ in terms of the Jack polynomials.

Proposition 3.3. We have

$$
z_{i} E_{\eta}(z)=\alpha d_{\eta}^{\prime} \sum_{\substack{I \subseteq\{1, \ldots, n\} \\ i \in I}} \frac{a_{I}^{(i)}\left(\frac{\overline{c_{I}(\eta)}}{\alpha}\right)}{d_{c_{I}(\eta)}^{\prime}} E_{c_{I}(\eta)}(z) .
$$

Proof. The nonsymmetric Jack polynomials $E_{\eta}$ with $|\eta|=d$ form a basis for the homogeneous polynomials of degree $d$. Hence we can write

$$
z_{i} E_{\eta}(z)=\sum_{|\mu|=|\eta|+1} c_{\mu} E_{\mu}(z)
$$


for coefficients $c_{\mu}$. Applying $\Psi$ to both sides of (3.19) and using the inversion formula (2.16) gives

$$
\tilde{Z}_{i} E_{\eta}^{*}(z)=\sum_{|\mu|=|\eta|+1} c_{\mu} E_{\mu}^{*}(z) .
$$

We now evaluate (3.20) at $z=\frac{\bar{\lambda}}{\alpha}$ where $|\lambda|=|\eta|+1$. It follows from the vanishing properties of the interpolated Jack polynomials and Proposition 3.2 that

$$
c_{\lambda}=\sum_{\substack{I \subseteq\{1, \ldots, n\} \\ i \in I, c_{I}(\eta)=\lambda}} \frac{a_{I}^{(i)}\left(\frac{\overline{c_{I}(\eta)}}{\alpha}\right) E_{\eta}^{*}\left(\frac{\bar{\eta}}{\alpha}\right)}{E_{c_{I}(\eta)}^{*}\left(\frac{\bar{c}_{I}(\eta)}{\alpha}\right)} .
$$

We obtain (3.18) by applying Proposition 3.1 to (3.21).

We can make two improvements to the formula (3.18). First, we can simplify the coefficient $a^{(i)}\left(\overline{\frac{c_{I}(\eta)}{\alpha}}\right)$ 1] Let

$$
\begin{aligned}
\tilde{B}_{I}(z):= & \left(\prod_{j=t_{s}+1}^{n} \frac{z_{t_{1}}+1-z_{j}-\frac{1}{\alpha}}{z_{t_{1}}+1-z_{j}}\right)\left(z_{t_{1}}+1+\frac{n-1}{\alpha}\right) \\
& \times\left(\prod_{j=1}^{t_{1}-1} \frac{z_{t_{1}}-z_{j}-\frac{1}{\alpha}}{z_{t_{1}}-z_{j}}\right)\left(\prod_{u=2}^{s} \prod_{j=t_{u-1}+1}^{t_{u}-1} \frac{z_{t_{u}}-z_{j}-\frac{1}{\alpha}}{z_{t_{u}}-z_{j}}\right), \\
\tilde{\chi}_{I}^{(i)}(z):= & \begin{cases}z_{i}-z_{t_{k+1}} & \text { if } i=t_{k}, k \in\{1,2,3, \ldots, s-1\}, \\
z_{i}-z_{t_{1}}-1 & \text { if } i=t_{s},\end{cases}
\end{aligned}
$$

where $I=\left\{t_{1}, \ldots, t_{s}\right\}, 1 \leq t_{1}<\cdots<t_{s} \leq n$. We have $\tilde{B}_{I}\left(\frac{\bar{\eta}}{\alpha}\right)=B_{I}\left(\frac{\overline{c_{I}(\eta)}}{\alpha}\right)$ and $\tilde{\chi}_{I}^{(i)}\left(\frac{\bar{\eta}}{\alpha}\right)=\chi_{I}^{(i)}\left(\frac{\overline{I_{I}(\eta)}}{\alpha}\right)$. Since $A_{I}\left(\frac{\bar{\eta}}{\alpha}\right)=A_{I}\left(\frac{\overline{c_{I}(\eta)}}{\alpha}\right)$, it follows that

$$
a_{I}^{(i)}\left(\frac{\overline{c_{I}(\eta)}}{\alpha}\right)=\tilde{\chi}_{I}^{(i)}\left(\frac{\bar{\eta}}{\alpha}\right) A_{I}\left(\frac{\bar{\eta}}{\alpha}\right) \tilde{B}_{I}\left(\frac{\bar{\eta}}{\alpha}\right) .
$$

Second, we can restrict the domain of summation in (3.18) by removing a number of vanishing terms. This allows us to give a unique decomposition of $z_{i} E_{\eta}$ in terms of the nonsymmetric Jack polynomials.

Proposition 3.4. Let $I:=\left\{t_{1}, \ldots, t_{s}\right\}$ with $1 \leq t_{1}<\cdots<t_{s} \leq n$ and $I \neq \emptyset$. We call I maximal with respect to $\eta$ iff:

i): $\eta_{j} \neq \eta_{t_{1}}, \quad j=1, \ldots, t_{1}-1$,

ii): $\eta_{j} \neq \eta_{t_{u}}, \quad j=t_{u-1}+1, \ldots, t_{u}-1$ and $u=2, \ldots, s$,

iii): $\eta_{j} \neq \eta_{t_{1}}+1, \quad j=t_{s}+1, \ldots, n$.

Let $\mathbb{J}_{\eta}$ be the set of non-empty subsets of $\{1, \ldots, n\}$ which are maximal with respect to $\eta$. The decomposition of $z_{i} E_{\eta}$ in terms of the nonsymmetric Jack polynomials is given by

$$
z_{i} E_{\eta}(z)=\alpha d_{\eta}^{\prime} \sum_{\substack{I \in \mathbb{J}_{\eta} \\ i \in I}} \frac{\tilde{\chi}_{I}^{(i)}\left(\frac{\bar{\eta}}{\alpha}\right) A_{I}\left(\frac{\bar{\eta}}{\alpha}\right) \tilde{B}_{I}\left(\frac{\bar{\eta}}{\alpha}\right)}{d_{c_{I}(\eta)}^{\prime}} E_{c_{I}(\eta)}(z) .
$$

\footnotetext{
${ }^{1}$ This simplification was pointed out to me by David McAnally.
} 
Proof. If $I$ is a non-empty subset of $\{1, \ldots, n\}$ with $I \notin \mathbb{J}_{\eta}$, then $\tilde{B}_{I}\left(\frac{\bar{\eta}}{\alpha}\right)=0$. Hence, we can restrict (3.18) to range over $\mathbb{J}_{\eta}$. Furthermore, if $I, I^{\prime} \in \mathbb{J}_{\eta}$ and $c_{I}(\eta)=c_{I^{\prime}}(\eta)$, then $I=I^{\prime}$. It follows that each Jack polynomial in the restricted sum only appears once.

\section{Generalised binomial coefficients}

The classical binomial coefficients

$$
\left(\begin{array}{l}
l \\
p
\end{array}\right):=\frac{l !}{(l-p) ! p !}
$$

occur in the identities

$$
e^{z} z^{p}=\sum_{l=p}^{\infty}\left(\begin{array}{l}
l \\
p
\end{array}\right) \frac{p !}{l !} z^{l}
$$

and

$$
(1+z)^{l}=\sum_{p=0}^{l}\left(\begin{array}{l}
l \\
p
\end{array}\right) z^{p}
$$

Rather than using (4.1) to define the binomial coefficients we could use either (4.2) or 4.3). Taking this latter perspective, we can define generalised binomial coefficients by an appropriate Jack polynomial generalisation of (4.2) or 44.3) 11 12. For $|\eta| \geq|\nu|$, we can define the generalised nonsymmetric binomial coefficients $\left(\begin{array}{l}\eta \\ \nu\end{array}\right)$ by

$$
\frac{E_{\eta}(1+z)}{E_{\eta}\left(1^{n}\right)}=\sum_{\nu|| \nu|\leq| \eta \mid}\left(\begin{array}{l}
\eta \\
\nu
\end{array}\right) \frac{E_{\nu}(z)}{E_{\nu}\left(1^{n}\right)} .
$$

This identity reduces to (4.3) when $n=1$. Using (4.4) to define $\left(\begin{array}{l}\eta \\ \nu\end{array}\right)$ we can derive the natural Jack polynomial generalisation of (4.2) (see e.g. 4])

$$
\frac{\alpha^{|\eta|}}{d_{\eta}^{\prime}} e^{e_{1}(z)} E_{\eta}(z)=\sum_{\nu|| \nu|\geq| \eta \mid} \frac{\alpha^{|\nu|}}{d_{\nu}^{\prime}}\left(\begin{array}{l}
\nu \\
\eta
\end{array}\right) E_{\nu}(z)
$$

where $e_{1}(z)$ is the first elementary symmetric function defined by (1.4). Alternatively, we may use (4.5) to define the generalised binomial coefficients and derive (4.4) as a consequence.

The generalised binomial coefficients are closely related to the interpolated Jack polynomials via the formula [12]

$$
\left(\begin{array}{l}
\eta \\
\nu
\end{array}\right)=\frac{E_{\nu}^{*}(\eta)}{E_{\nu}^{*}(\nu)}
$$

This formula generalises the classical identity

$$
\left(\begin{array}{l}
l \\
p
\end{array}\right)=\frac{(-1)^{p}(-l)_{p}}{(-1)^{p}(-p)_{p}}
$$

which relates the binomial coefficients $\left(\begin{array}{l}l \\ p\end{array}\right)$ to the classical shifted polynomials.

Using the decomposition of the product of $E_{\eta}(\eta)$ with $z_{i}$ we can give an evaluation of the generalised binomial coefficients for a restricted domain. We first require an analogue of (1.3) when $r=1$. 


\section{Proposition 4.1.}

$$
e_{1}(z) E_{\eta}(z)=-\alpha d_{\eta}^{\prime} \sum_{I \in \mathbb{J}_{\eta}} \frac{A_{I}\left(\frac{\bar{\eta}}{\alpha}\right) \tilde{B}_{I}\left(\frac{\bar{\eta}}{\alpha}\right)}{d_{c_{I}(\eta)}^{\prime}} E_{c_{I}(\eta)}(z) .
$$

Proof. It follows from (3.25) that

$$
\left(\sum_{i=1}^{n} z_{i}\right) E_{\eta}(z)=\alpha d_{\eta}^{\prime} \sum_{I \in \mathbb{J}_{\eta}}\left(\sum_{i \in I} \tilde{\chi}_{I}^{(i)}\left(\frac{\bar{\eta}}{\alpha}\right)\right) \frac{A_{I}\left(\frac{\bar{\eta}}{\alpha}\right) \tilde{B}_{I}\left(\frac{\bar{\eta}}{\alpha}\right)}{d_{c_{I}(\eta)}^{\prime}} E_{c_{I}(\eta)}(z) .
$$

The identity (4.8) follows since $e_{1}(z)=\sum_{i=1}^{n} z_{i}$ and $\sum_{i \in I} \tilde{\chi}_{I}^{(i)}\left(\frac{\bar{\eta}}{\alpha}\right)=-1$.

Corollary 4.2. Suppose $|\mu|=|\eta|+1$. Then

$$
\left(\begin{array}{c}
\mu \\
\eta
\end{array}\right)=-A_{I}\left(\frac{\bar{\eta}}{\alpha}\right) \tilde{B}_{I}\left(\frac{\bar{\eta}}{\alpha}\right),
$$

where $\mu=c_{I}(\eta)$. If there is no I such that $\mu=c_{I}(\eta)$, then $\left(\begin{array}{l}\mu \\ \eta\end{array}\right)=0$.

Proof. It follows from 4.5 that

$$
e_{1}(z) E_{\eta}(z)=\alpha d_{\eta}^{\prime} \sum_{|\nu|=|\eta|+1} \frac{1}{d_{\nu}^{\prime}}\left(\begin{array}{l}
\nu \\
\eta
\end{array}\right) E_{\nu}(z) .
$$

Comparison with (4.8) gives (4.10).

\section{REFERENCES}

[1] T.H. Baker and P.J. Forrester, Nonsymmetric Jack polynomials and integral kernels, Duke Math. J. 95 (1998), 1-50. MR 2000b:33006

[2] _ Symmetric Jack polynomials from nonsymmetric theory, Ann. Comb. 3 (1999), 159170. MR 2002e:33021

[3] I. Cherednik, Nonsymmetric Macdonald polynomials, Int. Math. Res. Not. 10 (1995), 483515. MR 97f:33032

[4] P. Forrester, Log-gases and random matrices, in preparation.

[5] F. Knop, Symmetric and non-symmetric quantum Capelli polynomials, Comment. Math. Helv. 72 (1997), 84-100. MR 98m:05204

[6] F. Knop and S. Sahi, Difference equations and symmetric polynomials defined by their zeros, Int. Math. Res. Not. 10 (1996), 473-486. MR 99d:05086

[7] _ A recursion and combinatorial formula for the Jack polynomials, Invent. Math. 128 (1997), 9-22. MR 98k:33040

[8] E. Opdam, Harmonic analysis for certain representations of graded Hecke algebras, Acta. Math. 175 (1995), 75-121. MR 98f:33025

[9] S. Sahi, The spectrum of certain invariant differential operators associated to a Hermitian symmetric space, Lie Theory and Geometry: in honour of B. Konstant (V. Gaillemin, J.L. Brylinski, R. Brylinski and V. Kac, eds.), Prog. Math, vol. 123, Birkhauser, Boston, 1994, pp. 569-576. MR 96d:43013

[10] - Interpolation, integrality, and a generalization of Macdonald's polynomials, Int. Math. Res. Not. 10 (1996), 457-471. MR 99j:05189b

[11] _ A new scalar product for the non-symmetric Jack polynomials, Int. Math. Res. Not. 20 (1996), 997-1004. MR 98g:05154

[12] _ The binomial formula for nonsymmetric Macdonald polynomials, Duke. Math. J. 94 (1998), 465-477. MR 99k:33041 
[13] R. P. Stanley, Some combinatorial properties of Jack symmetric functions, Advances in Math. 77 (1989), 76-115. MR 90g:05020

[14] G. Szegö, Orthogonal polynomials, 4th ed., American Mathematical Society, Providence R.I., 1975. MR 51:8724

School of Humanities, Australian National University, Canberra, 0200, Australia

E-mail address: Dan.Marshall@anu.edu.au 